\title{
An unexpected cause of persistent bacteraemia and portomesenteric venous gas
}

\author{
A. Loobuyck ${ }^{1}$, G. Vermeersch ${ }^{2}$, M. D’Hondt ${ }^{3}$, T. Billiet ${ }^{4}$
}

(1) Faculty of Medicine and Health Sciences, University of Ghent, Ghent, Belgium \& Department of Internal Medicine, AZ Groeninge, Kortrijk, Belgium ; (2) Faculty of Medicine and Health Sciences, Catholic University of Leuven, Leuven, Belgium \& Clinical Internship at the Department of Internal Medicine, AZ Groeninge, Kortrijk, Belgium ; (3) Department of Abdominal Surgery, AZ Groeninge, Kortrijk, Belgium ; (4) Department of Gastroenterology and Hepatology, AZ Groeninge, Kortrijk, Belgium.

\begin{abstract}
We report the case of a 59-year old man with portomesenteric venous gas (PMVG) due to inferior mesenteric vein fistulization caused by sigmoid diverticulitis with an unusual evolution. The patient initially presented with classic symptoms of lower abdominal pain and fever. Diagnosis of uncomplicated sigmoid diverticulitis was confirmed on computed tomography (CT) for which intravenous antibiotics were initiated. Hemocultures were positive for omnisensitive Escherichia Coli, but despite adequate intravenous antibiotic therapy, episodes of bacteraemia persisted and hemocultures remained positive. Repeat CT scan demonstrated regression of inflammation without signs of abcedation or perforation consistent with clinical findings. Endocarditis was excluded with a normal transoesophageal echocardiography. Finally, positron emission tomography-computed tomography (PET-CT) suspected a colovenous fistula and the presence of PMVG. The patient was successfully treated with laparoscopic sigmoidectomy. This case report summarises the diagnostic pathway and aims for higher awareness of non-ischemic PMVG causes. (Acta gastroenterol. belg., 2021, 84, 375-377).
\end{abstract}

Key words : Diverticulitis, fistulization, portomesenteric venous gas

Abbreviations : PMVG : portomesenteric venous gas ; CT : Computed Tomography ; PET-CT : Positron emission tomography-computed tomography ; IV : intravenous ; MRI : Magnetic Resonance Imaging.

\section{Introduction}

Portomesenteric venous gas (PMVG) caused by fistulization is a rare complication of diverticulitis. As intestinal ischemia/gangrene is the most common cause of PMVG, it is historically associated with high mortality rates (1-5). Improved imaging techniques resulted in the identification of more non-ischemic causes with lower mortality rates (6). More awareness on these nonischemic causes of PMVG is necessary in order to reduce time-to-diagnosis in patients presenting with nonspecific symptoms.

\section{Case report}

A 59-year old Belgian male presented at the emergency department with several days of intense pain located in the suprapubic and left fossa region, accompanied with fever since a few hours. Medical history was limited to urinary retention due to benign prostatic hypertrophy for which silodosine $(8 \mathrm{mg}$ ) was prescribed. At the emergency department the patient was subfebrile $\left(37.8^{\circ} \mathrm{C}\right)$ and had mild tachycardia with normal blood pressure $(151 / 87 \mathrm{mmHg})$. During clinical investigation left fossa and suprapubic tenderness and guarding were present. Biochemical analysis showed a white blood cell count of $4.72 \times 10^{9} / \mathrm{L}$ (ref. range: $4.20-9.80 \times 10^{9} / \mathrm{L}$ ), an elevated total bilirubin level of $2.61 \mathrm{mg} / \mathrm{dL}$ (ref. range: $<1.20 \mathrm{mg} / \mathrm{dL}$ ), mainly indirect, and a C-reactive protein (CRP) level of $20 \mathrm{mg} / \mathrm{L}$ (ref. range: $<5.0 \mathrm{mg} / \mathrm{L}$ ), which raised to $266 \mathrm{mg} / \mathrm{L}$ the day after. Arterial blood gas analysis showed a normal $\mathrm{pH}$ and a raised lactate of 3.3 $\mathrm{mmol} / \mathrm{L}$ (ref. range: 0.5-2.2 $\mathrm{mmol} / \mathrm{L}$ ). Urine cultures were negative, while obtained hemocultures resulted positive for Escherichia Coli, sensitive to amoxicillin/clavulanic acid and piperacillin/tazobactam. Contrast enhanced abdominal computed tomography (CT) demonstrated uncomplicated sigmoidal diverticulitis, together with multiple large biliary cysts in the liver and a solitary cyst in the right kidney. Empiric intravenous (IV) antibiotic therapy (amoxicillin/clavulanic acid and single dose of amikacin) was initiated on day of admission. Despite sensitivity to penicillins, recurrent febrile episodes with heavy shivering developed after 48 hours and persisted for several days onwards. Hemocultures remained positive for Escherichia Coli, for which antibiotic therapy was switched to IV piperacillin/tazobactam. Repeat abdominal imaging with CT scan showed a decrease of inflammation around the sigmoid and no signs of complication: no abcedation or perforation. This was consistent with clinical evolution: there was less abdominal pain and tenderness. Nevertheless, despite therapy intensification, bacteraemia persisted so further investigation for an additional focus of bacteraemia was required.

There were no clinical clues of additional foci of infection (no tooth pain, no headaches, no joint pain or skin rash). Consequently, endocarditis was excluded by transthoracic and transoesophageal echocardiography and normal fundoscopic examination. Secondly, magnetic resonance imaging (MRI) of the liver confirmed the presence of multiple benign biliary cysts without arguments for inflammation. Ultrasound guided percutaneous aspiration of the largest cyst revealed clear fluid

\footnotetext{
Correspondence to: Astrid Loobuyck. Rendekensstraat 82, 9070 Heusden (Destelbergen), Belgium.

Email : Astrid.Loobuyck@UGent.be

Submission date : 05/07/2020

Acceptance date : 22/08/2020
} 


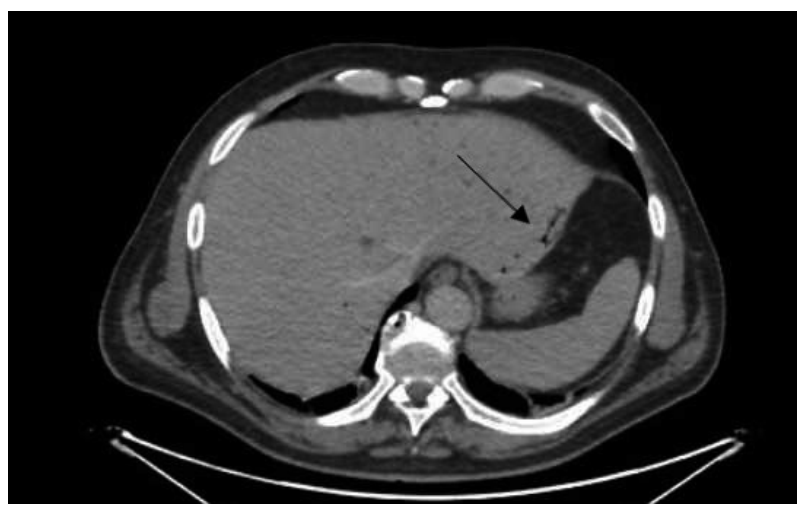

Figure 1. - CT image of the PET-CT scan on day seven of hospitalisation showing the portomesenteric venous gas in the hepatic portal veins (black arrow).

without infection. Finally, because of the persisting bacteraemia, positron emission tomography with $\mathrm{CT}$ (PET-CT) was performed and identified the presence of portomesenteric venous gas (PMVG) (ex. Fig. 1). with a suspected colovenous fistula, located at the sigmoid region of the inflamed diverticulum (ex. Fig. 2). Surgical resection of the fistula by laparoscopic sigmoidectomy was executed nine days after administration. Primary end-to-end anastomosis was preferred instead of the Hartmann procedure. The patient recovered well and was discharged free of symptoms. Pathologic examination of the resected specimen showed benign sigmoid diverticulitis. A follow-up colonoscopy two months later only showed diverticular disease.

\section{Discussion}

Of the patients with acute diverticulitis 35\% develop complications, with fistula being less common (9-14\%), mostly colovesical fistula followed by coloenteric or colouterine fistula (7). In our case, no complications were seen on CT the day of presentation, nor on the repeat CT scan three days later. The PET-CT on day seven of hospitalisation revealed the presence of a colovenous fistula with portomesenteric venous gas, while this was not seen on the MRI liver one day earlier. Intermittent PMVG has been reported before and is thought to be related to the intra-abdominal pressure, pushing a jet of gas, feces and pus into the portomesenteric circulation when rising (6).

Overall, intestinal ischemia/gangrene is identified as the most common mechanism of onset in PMVG, with prevalence ranging from approximately $40-70 \%$. Historically, reported PMVG mortality rates were estimated at approximately $80 \%$ demanding urgent laparotomy. However, due to improved imaging techniques, allowing the detection of smaller amounts of gas, mortality rates declined to less than $50 \%(1-6,8-10)$. Additionally, improved imaging techniques also led to the identification of other, non-ischemic aetiologies of

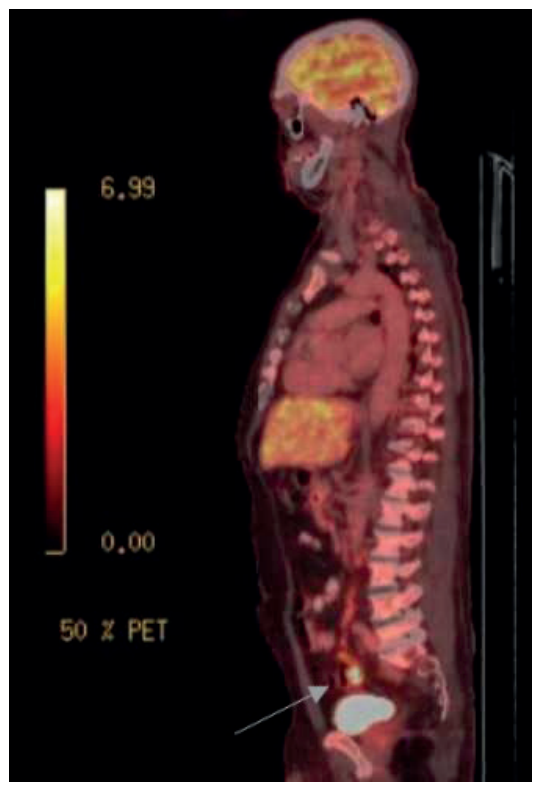

Figure 2. - PET-CT scan on day seven of hospitalisation showing the inflamed diverticulum at the sigmoid region (grey arrow) with the suspected colovenous fistula above.

PMVG such as diverticulitis, ulcerative colitis, gastric ulcers and Crohn's disease $(3,8)$. Currently, few data on these non-ischemic PMVG causes are available. A review by Sellner et al. identified complicated diverticulitis as the second most common cause of PMVG after intestinal ischemia. Septic thrombophlebitis of the inferior mesenteric vein $(45 \%)$ or direct colovenous communication, due to necrotising vasculitis in the wall of a present intramesocolic abscess (55\%), are reported as etiological mechanisms in complicated diverticulitis $(6,9)$. On the other hand, Daneshmand et al. reported bowel obstruction $(17 \%)$ as the most frequent nonischemic cause out of 164 PMVG cases. Diverticulitis was reported in approximately $2 \%$ of all PMVG cases (2). In our case no thrombophlebitis or intramesocolic abscess was identified by preceding medical imaging techniques. However, the presence of a mesocolic abscess was revealed during laparoscopic exploration. Besides focal adipose infiltration, post-hoc analysis of the obtained radiological images did not discover any signs of abcedation in the left fossa region.

This case report supports the need for further etiological identification and classification in PMVG. Clinicians should be aware of the diversity of PMVG causes and the possible complications of an acute diverticulitis (10). Raising more awareness on these non-ischemic causes of PMVG will result in further streamlining the diagnostic process and which hopefully will lead to faster adequate therapy aiming for reduced mortality rates.

\section{Conflicts of interest}

The authors declare that they have no conflicts of interest concerning this article. 


\section{References}

1. PARAN H., EPSTEIN T., GUTMAN M., FEINBERG MS., ZISSIN R. Mesenteric and portal vein gas: computerized tomography findings and clinical significance. Dig. Surg., 2003, 20 (2) : 127-132.

2. DANESHMAND A., PARYS S., RAO S., WANTANABE Y., SIEUNARINE K. Portal venous gas: different aetiologies and their respective outcomes. ANZ J. Surg., 2020. DOI : 10.1111/ans.15795.

3. KINOSHITA H., SHINOZAKI M., TANIMURA H., UMEMOTO Y., SAKAGUHI S., TAKIFUJI K., et al. Clinical features and management of hepatic portal venous gas: Four case reports and cumulative review of the literature. Arch. Surg., 2001, 136 (12) : 1410-1414.

4. HEYE T., BERNHARD M., MEHRABI A., KAUCZOR HU., HOSCH W. Portomesenteric venous gas: Is gas distribution linked to etiology and outcome? Eur. J. Radiol., 2012, 81 (12) : 3862-3869.

5. HUSSAIN A., MAHMOOD H., EL-HASANI S. Portal venous gas in emergency surgery. World J. Emerg. Surg., 2008, 3 (21). DOI : 10.1186/17497922-3-21
6. SELLNER F., SOBHIAN B., BAUR M., SELLNER S., HORVATH B., MOSTEGEL M., Intermittent hepatic portal vein gas complicating diverticulitis - A case report and literature review. Int. J. Colorectal. Dis., 2007, 22 (11) : 1395-1399.

7. SESSA B., GALLUZZO M., IANNIELlO S., PINTO A., TRINCI M. MIELE V. Acute perforated diverticulitis: Assessment with multidetector computed tomography. Semin. Ultrasound CT, 2016, 37 : 37-48.

8. TREYAUD MO., DURAN R., ZINS M., KNEBEL JF., MEULI RA., SCHMIDT S. Clinical significance of pneumatosis intestinalis - correlation of MDCT - findings with treatment and outcome. Eur. Radiol., 2017, 27 (1) : 70-79.

9. ZIELKE A., HASSE C., NIES C., ROTHMUND M. Hepatic-portal venous gas in acute colonic diverticulitis. Surg. Endosc., 1998, 12 (3) : 278-280.

10. YATES TAE., PASIPANODYA A., SATHYANARAYANA SA. Portomesenteric venous gas secondary to acute diverticulitis colovenous fistula. Am. Surg., 2018, 84 (4) : E123-E124. 